\title{
Persistence of a Liver Metabolic Zonation in Extra-Hepatic Biliary Atresia Cirrhotic Livers
}

\author{
E. M. SOKAL, E. COLlETte, AND J. P. BUTS
}

Laboratory of Pediatric Hepatology [E.M.S., E.C.] and Pediatric Gastroenterology [J.P.B.], Unit of Gastroenterology and Nutrition, Catholic University of Louvain St LUC Hospital, Brussels, Belgium

\begin{abstract}
To evaluate whether liver metabolic zonation persists in human biliary cirrhosis, we used quantitative cytochemistry to measure activities of glucose 6 phosphatase (G6P) and NADPH dehydrogenase (ND) in hepatocytes situated in different zones of liver cirrhotic nodules. Liver specimens were obtained from 13 children with extrahepatic biliary atresia with compensated cirrhosis. Activity and distribution were compared with zonal activities measured in 17 control human liver specimens obtained during reduction hepatectomies for orthotopic liver transplantation. In normal human liver, G6P was 1.86 times more active in the periportal than in the perivenular zone. On the contrary, ND activity was lower in the periportal zone (63\% of perivenular activity). A metabolic zonation persisted in extra-hepatic biliary atresia with compensated cirrhosis. G6P activity was 1.56 times greater at the nodule periphery than at the nodule center, whereas ND activity was lower at the periphery ( $75 \%$ of nodule center activity). This metabolic zonation is the opposite of that observed in animal toxic $\left(\mathrm{CCl}_{4}\right)$ cirrhosis, in which greater $\mathrm{G6P}$ activity is observed at the nodule center and greater ND activity at its periphery. This confirms our previous hypothesis that the type of cirrhotic metabolic zonation may depend on the site of initial liver damage. (Pediatr Res 30: 286-289, 1991)
\end{abstract}

\section{Abbreviations}

G6P, glucose 6 phosphatase (EC 3.1.3.9)

ND, NADPH dehydrogenase (EC 1.6.99.1)

EHBA, extra-hepatic biliary atresia

MIE, mean integrated extinction

$\mathrm{NC}$, nodule center

NP, nodule periphery

The metabolic activity of human and mammal hepatocytes differs according to their localization inside the liver acinus. This functional heterogeneity of hepatocytes, called liver cell zonation, is also influenced by changes in extra-cellular fluid composition and seems to play a major role in maintaining the homeostatic balance (1-4). Various methods have been described to study this zonation. Among these, quantitative cytochemistry is reliable and can easily be applied to study biopsy samples (5). We recently showed that liver cell heterogeneity may persist after extensive pathologic changes leading to cirrhosis in growing rats intoxicated with carbon tetrachloride (6). A periportal-like metabolism

Received October 31, 1990; accepted April 15, 1991.

Correspondence and reprint requests: Dr. Etienne M. Sokal, Département de Pédiatrie 1301, Unité de Gastro-hépatologie pédiatrique, 10 avenue Hippocrate, 1200 Brussels, Belgium.

Supported by Research grants no. 9451288 and 3451589 from the "Fonds National de la Recherche Médicale,” Brussels, Belgium. was observed at the center of cirrhotic nodules and a perivenularlike activity at their periphery (6). However, these changes could be specific to the animal species and to the model of cirrhosis, and a different zonation might be observed in a human biliary type cirrhosis. To verify this hypothesis, we used quantitative cytochemistry (5) to measure zonal activities of G6P and ND in cirrhotic livers from children with EHBA. Our aim was to evaluate adaptive changes of zonal enzyme activities in the cirrhotic nodules from this human biliary cirrhosis as compared with normal human liver. The two enzymes assayed in this study have a different zonation in normal human liver, despite the fact that both are situated in the endoplasmic reticulum (5). G6P, more active in the periportal zone, is a key enzyme of gluconeogenesis, whereas ND is involved mainly in hormones, drugs, and xenobiotics metabolism (1-5).

\section{MATERIALS AND METHODS}

For the present study, liver specimens from five girls and eight boys $(n=13)$ were obtained. They had histologically proven cirrhosis due to EHBA. All of them were in Child's class A. At the time of liver sampling, the mean age of the patients was 21 mo (range 6 to $45 \mathrm{mo}$ ). The liver tissue was obtained either by percutaneous biopsy, using the Menghini technique (Hepafix needle 1.4, Braun, Melsungen, Germany), or during laparotomy for orthothopic liver transplantation, before devascularization of the liver. The biopsy was immediately frozen in cryomatrix compound (HVL, Brussels, Belgium), cooled on a metallic cylinder immersed in liquid nitrogen. It was then stored in liquid nitrogen until enzymatic assays were performed, within 1 wk from sampling. A piece of liver was also fixed in Bouin solution for histologic analysis (Hemalun eosin staining).

Controls. Seventeen samples of normal human livers ( 10 males and 7 females, mean age $15 \mathrm{y}$, range $7-33 \mathrm{y}$ ), were obtained from donor livers during reduction hepatectomies of grafts for transplantation (7). These samples were frozen and stored following the same procedure as the livers from cirrhotic patients. Before sampling, the liver grafts had been perfused and cooled for a mean of $13 \mathrm{~h}$ (range 9-16 h) in the University of Wisconsin Solution (7). Liver function tests of donors were normal.

Ten-micron frozen sections were cut at $-30^{\circ} \mathrm{C}$ in a Shandon cryostat (HVL, Brussels, Belgium) and stored at this temperature for a maximum of $2 \mathrm{~h}$ before enzymatic assays were performed (5)

Cytochemical Assays. The sections were defrosted and immediately incubated as previously described $(5,6)$ in the following reaction media.

G6P. Acetate buffer $0.1 \mathrm{mmol} / \mathrm{L}, \mathrm{pH} 6.5$; glucose-6-phosphate $2.1 \mathrm{mmol} / \mathrm{L} ; \mathrm{PBNO}_{3} 2.4 \mathrm{mmol} / \mathrm{L}$ was used $(3,4,6)$. The reaction was carried out in a Coplin jar at $37^{\circ} \mathrm{C}$ and stopped after $6 \mathrm{~min}$ by washing the slides in distilled water. The slides were then immersed in sulfide water for $10 \mathrm{~s}$ to convert the lead phosphate precipitate into lead sulfide. For each section, a control reaction 
was also performed on a serial section, but without adding substrate in the medium (nonsubstrate section).

$N D$. Incubation medium was glycyl glycine buffer $50 \mathrm{mmol} /$ $\mathrm{L}$, pH 7.9; polyvinyl alcohol, 20\%; $\mathrm{NADPH}_{2}, 3 \mathrm{mmol} / \mathrm{L}$; nitroblue tetrazolium, $4.9 \mathrm{mmol} / \mathrm{L}(5,6,8,9)$. Incubations were performed at $37^{\circ} \mathrm{C}$ in plastic rings for $8 \mathrm{~min}$ and the reaction was stopped by washing in distilled water. A nonsubstrate section was also performed for each specimen.

After incubation, the sections were dried, mounted in Aquamount medium (Kindler GMBH, Freiburg, Germany), and stored in the dark until reading of densities.

The density of the colored end-product of reaction was measured by microspectrophotometry using a Vickers M 85 A scanning and integrating microdensitometer $(5,11,12)$ (Vickers Instruments, York, UK) with the following setting: Mask A3, spot size $0.5 \mu \mathrm{m}$, magnification $\times 25$. With this setting, the area covered by the mask corresponded to two to three hepatocytes. In normal livers, 10 readings were made at random in three to five different periportal zones and in three to five different perivenular zones in each section. In cirrhotic livers, 10 readings were made along fibrous septa (NP) and 10 in the center of cirrhotic nodules (NC). Densities were also measured in the corresponding nonsubstrate sections, and these values were deducted from the density measured in the substrate section. The mean integrated density measured was converted into MIE $U$ by comparing the mean integrated density of the specimen (minus mean integrated density of the nonsubstrate section) with the density of a filter of known extinction value equal to 1 . MIE $U$ were multiplied by 100 to avoid decimals $($ MIE $\times 100)(5)$. MIE values of the 13 patients and 17 controls are expressed as mean $\pm \mathrm{SD}$.

The metabolic zonation was expressed by the ratio of periportal to perivenular activity in normal livers and by the ratio of NP to NC activity in cirrhotic livers.

Mean zonal activities were compared by the $t$ test, and differences were considered as significant if $p$ was less than 0.01 .

Microphotographs were taken using a Ricoh camera and Kodak 100 ASA black and white films.

\section{RESULTS}

Light microscopy. In normal human livers, a heterogeneous pattern of enzyme activity was visible on light microscopy examination. G6P activity was higher in the periportal zone and ND activity was higher in the perivenular zone, as previously observed (5). In cirrhotic livers, G6P activity was more intense at the periphery of the cirrhotic nodules (Fig. 1), whereas ND

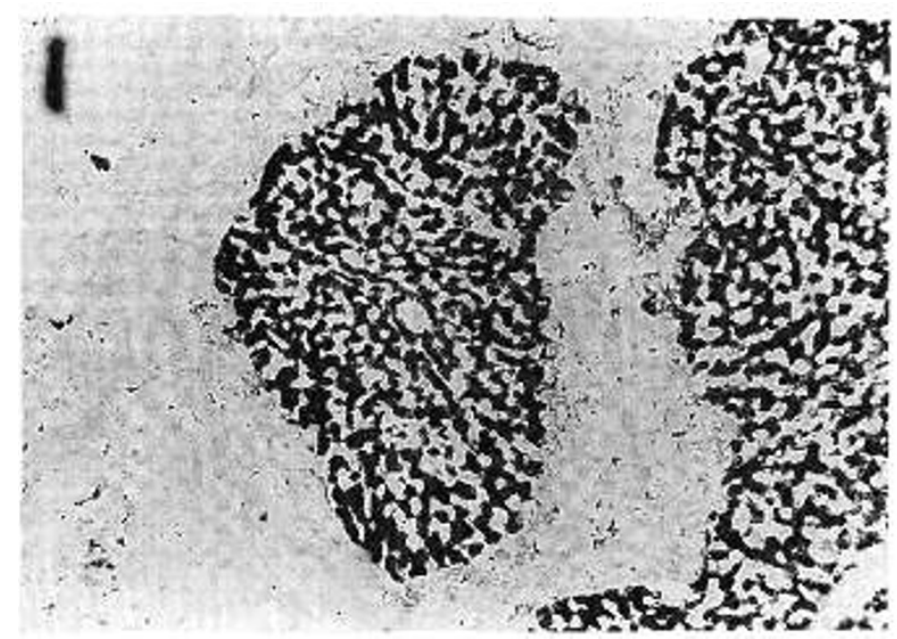

Fig. 1. Cirrhotic liver section, original magnification $\times 100$. The endproduct of G6P cytochemical reaction (lead sulfide) precipitates at the site of production. Activity is greater at the periphery of the cirrhotic nodule. Density is quantified by microdensitometry.

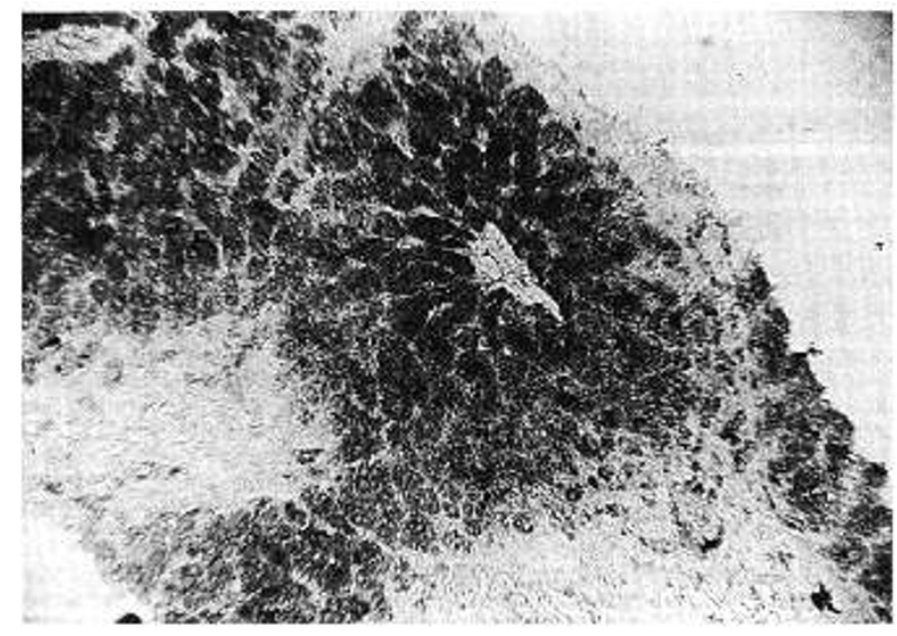

Fig. 2. Cirrhotic liver section, original magnification $\times 100$. The endproduct of NADPH dehydrogenase reaction is nitro blue tetrazolium formazan. It precipitates at the site of production, showing greater activity of this enzyme at the center of the cirrhotic nodule. Density of the precipitate is measured by microdensitometry.

activity was more intense at NC (Fig. 2). This cirrhotic liver cellular heterogeneity showed a pattern of "nodular zonation" that was quantified by microdensitometric measurements of the end-product of reaction.

Density measurements. The zonal enzyme activities measured in both normal (periportal and perivenular) and cirrhotic (NP and NC) livers are detailed in Figures 3 (G6P) and 4 (ND).

G6P (Fig. 3). In controls, activity was 1.86 times more active in the periportal zone (MIE $55.6 \pm 10.9$ ) than in the perivenular zone (MIE $30.2 \pm 12 ; p<0.01$ ). In cirrhotic livers, activity at the periphery of the cirrhotic nodule (MIE $84.8 \pm 17.8$ ) was 1.56 times higher than at the center (MIE $53.7 \pm 20.2 ; p<0.01$ ). In addition, G6P was more active in cirrhotic liver cells than in normal human liver cells: NP activity was 1.53 times greater than control periportal activity, and NC activity was 1.73 times greater than control perivenular activity (Fig. 3) $(p<0.01)$.

NADPH dehydrogenase (Fig. 4). In controls, ND periportal activity (MIE $46.2 \pm 10.9$ ) was $63 \%$ of the perivenular activity (MIE 73.1 $\pm 15.8 ; p<0.01$ ). In cirrhotic livers, activity at the NP (MIE $54.5 \pm 10.8$ ) was $75 \%$ of NC activity (MIE $73.5 \pm$ $10.7 ; p<0.01)$. When activities of cirrhotic livers were compared with controls, similar values were found between activity at the center of the cirrhotic nodules and activity in the perivenular zone of the control livers. Enzyme activity was also similar between the periphery of the cirrhotic nodules and the periportal zone of the control livers.

\section{DISCUSSION}

There is so far no report of the persistence of a liver metabolic zonation in human cirrhosis. This liver cell heterogeneity is known to play a major role in most liver metabolic pathways, and, as a result, for the final composition of the blood leaving the liver (1-4). Heterogenous function depends not only on acinar localization, but also from various environmental factors that may modify the balance between periportal and perivenular activities of the same enzyme (3). Metabolic zonation has been shown to persist in animal experimental cirrhosis, with progressive adaptation of liver cell heterogeneity to the new architecture of the cirrhotic liver $(6,13,14)$. From a previous study in a rat model of carbon-tetrachloride-induced cirrhosis, we suggested that a different type of "cirrhotic zonation" could occur in various pathologic situations, depending on the site of the initial liver damage (6). If our assumption is correct, a reverse cirrhotic nodular zonation should be observed in a biliary type cirrhosis 


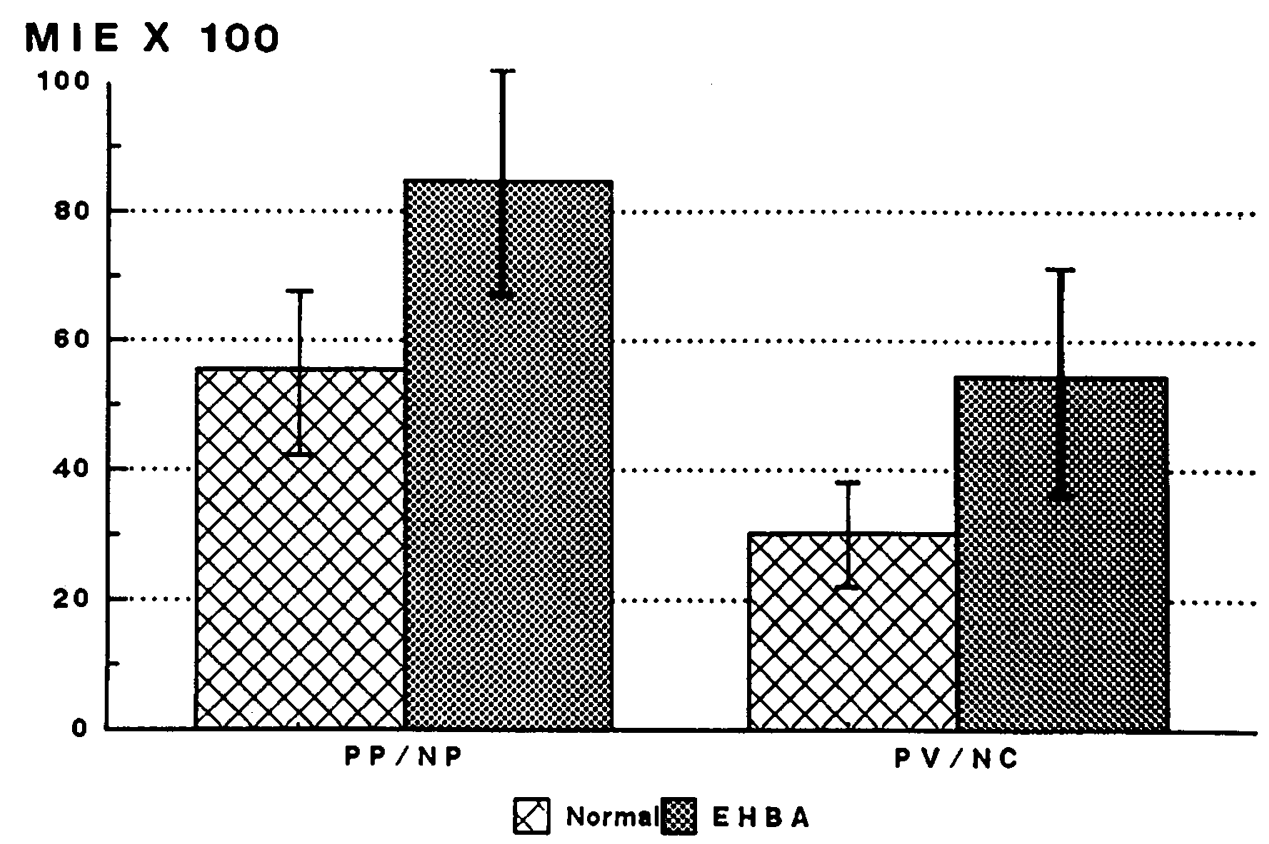

Fig. 3. Mean activities of G6P in 17 normal livers (hatched columns) and in 13 EHBA cirrhotic livers (screened columns). Activity is expressed as MIE $U \times 100$. Vertical lines express SD. $P P$, periportal activity in normal liver; $P V$, perivenular activity in normal liver; $N P$, NP activity in cirrhotic liver; $N C, \mathrm{NC}$ activity in cirrhotic livers.

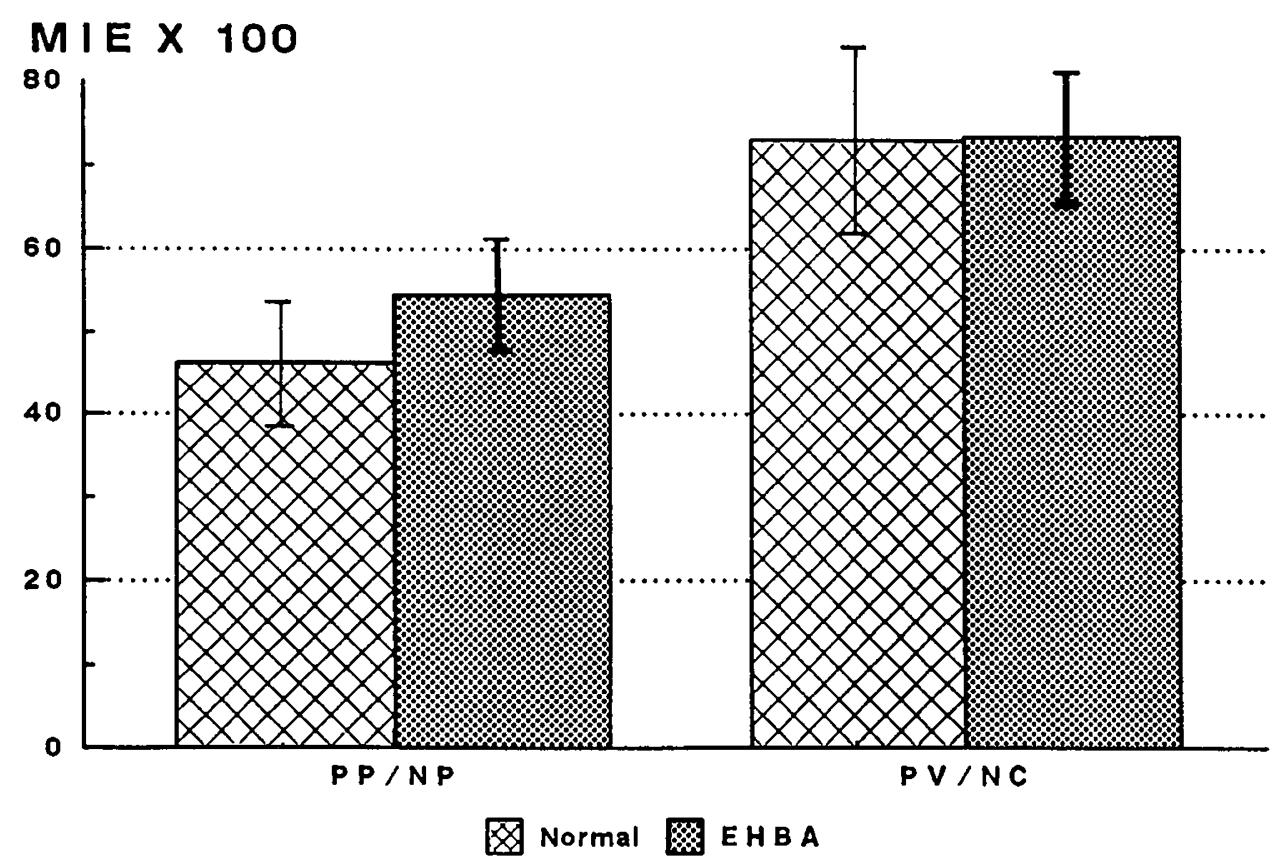

Fig. 4. Mean activity of NADPH dehydrogenase in 17 normal livers (hatched columns) and in 13 EHBA cirrhotic livers (screened columns). Activity is expressed as MIE $U \times 100$. Vertical lines express SD. $P P$, periportal activity in normal liver; $P V$, perivenular activity in normal liver; $N P$, $\mathrm{NP}$ activity in cirrhotic liver; $N C, \mathrm{NC}$ activity in cirrhotic livers.

(initial damage periportal) as compared with a toxic cirrhosis (perivenular damage) (6).

The present study provides evidence for this hypothesis. Indeed, the activity of G6P was found to be higher at the periphery of the cirrhotic nodules, suggesting that this zone contains the afferent vessels. This was further confirmed by the distribution of $\mathrm{ND}$, which was more active at the $\mathrm{NC}$, suggesting that this zone contains the efferent vessels. Because both enzymes are located in the same subcellular organelles, it appears unlikely that this zonation is due to local differences in microsome concentrations (15). In cirrhosis caused by EHBA, lesions are initially observed around the portal tracts and, subsequently, porto-portal bridging septa dissect the liver parenchyma. There- fore, one can suppose that these septa surround a perivenular zone, resulting in a cirrhotic nodule showing the zonal enzyme distribution observed here. Conversely, in toxic hepatitis, lesions are initially observed in the perivenular zone, and the bridging septa tend to surround a portal tract (16), which could result in a cirrhotic nodule with an inverse enzyme distribution. However, because this comparison is based on data obtained from patients with biliary cirrhosis and from an animal model of experimental $\left(\mathrm{CCl}_{4}\right)$ cirrhosis, additional comparative studies in humans are necessary before a firm conclusion can be drawn. For instance, it would be of particular interest to compare the metabolic zonation in human alcoholic cirrhosis with that of primary biliary cirrhosis. 
In addition, the present study provides interesting information on the zonation of G6P and ND activities in normal human livers, confirming in this larger group our preliminary observations (5). In this study, G6P activity was markedly increased in cirrhotic livers compared with normal human livers. These activities may vary depending on the physiologic status at the time of sampling $(1,3,4)$. Also, it must be stressed that our patients were younger than the donors, and G6P activity has been shown to be higher in young animals compared to adults (14). In addition, G6P is mainly involved in gluconeogenesis, and the higher activity observed in livers from cirrhotic children may reflect their higher catabolism, resulting in an increased demand of free glucose production for peripheral tissue use $(1,3,4,14)$. Other factors may play a role, such as preservation of the donor liver in University of Wisconsin Solution, whose composition may influence enzyme activities. ND activity was similar in patients and controls, but for the same reasons as stated with regard to G6P activity, no precise conclusion can be drawn from this observation $(1,3,4)$.

In summary, the present study demonstrates that a metabolic zonation of G6P and ND activities persists in human EHBA cirrhosis. The type of enzyme activity distribution observed suggests that afferent vessels are situated in the fibrous septa, at the periphery of the nodules, and that efferent vessels are located at their center. This is exactly the opposite of the pattern observed previously in an animal model of toxic $\mathrm{CCl}_{4}$ cirrhosis (6). It reinforces the hypothesis that the metabolism at the periphery of the cirrhotic nodule corresponds to the site of the initial liver damage, which is periportal in EHBA.

\section{REFERENCES}

1. Gumucio J, Chianale J 1988 Liver cell heterogeneity and liver function. In Arias IM, Jakoby WB, Popper H, Schachter D, Shafritz DA (eds) The Liver: Biology and Pathobiology. Raven Press, New York, pp 931-947
2. Katz NR 1989 Methods for the study of liver cell heterogeneity. Histochem J 21:517-529

3. Jungermann K, Katz N 1989 Functional specialization of different hepatocyte populations. Physiol Rev 69:708-764

4. Gumucio J 1989 Hepatocyte heterogeneity: the coming of age. From the description of a biological curiosity to a partial understanding of its physiological meaning and regulation. Hepatology 9:154-160

5. Sokal EM, Trivedi P, Cheeseman P, Portmann B, Mowat AP 1989 The application of quantitative cytochemistry to study the acinar distribution of enzymatic activities in human liver biopsy specimens. J Hepatol 9:42-48

6. Sokal EM, Trivedi P, Portmann B, Mowat AP 1990 Adaptive changes of metabolic zonation during the development of cirrhosis in growing rats. Gastroenterology 99:785-792

7. Otte JB, de Ville de Goyet J, Sokal EM 1989 Size reduction of the donor liver is a safe way to alleviate the shortage of size-matched organs in pediatric liver transplantation. Ann Surg 211:146-157

8. Teutsch HF 1978 Improved method for histochemical demonstration of glucose-6-phosphatase activity. Histochemistry 57:107-117

9. Chayen J, Bitensky L, Butcher R 1973 Practical Histochemistry. Wiley, London.

10. Altman F 1980 Tissue stabilizers methods in histochemistry. ln: Trends in Enzyme Histochemistry and Cytochemistry. Ciba Symposium 73. Excerpta Medica, Amsterdam, pp 81-93

11. Stoward P 1980 Criteria for the validation of quantitative histochemical enzyme techniques. In: Trends in Enzyme Histochemistry and Cytochemistry. Ciba Symposium 73. Excerpta Medica, Amsterdam, pp 11-27

12. Goldstein D 1978 lntegrating microdensitometry. In: Meek G, Gyder H (eds) Analytical Methods in Microscopy. Histochemistry 57:107-117

I3. Nuber R, Teutsch H, Sasse D 1980 Metabolic zonation in thioacetamide induced liver cirrhosis. Histochemistry 69:277-288

14. Sokal EM, Trivedi P, Portmann B, Mowat AP 1989 Developmental changes in the intra-acinar distribution of succinate dehydrogenase, glutamate dehydrogenase, glucose 6 phosphatase and NADPH dehydrogenase in the rat liver. J Pediatr Gastroenterol Nutr 8:522-527

15. Pette D, Wimmer Z 1980 Microphotometric determination of enzyme activities in cryostat sections by the gel film technique. In: Trends in Enzyme Histochemistry and Cytochemistry. Ciba Symposium 73. Excerpta Medica, Amsterdam, pp 121-130

16. Altermman K 1954 Studies in fibrosis of the liver induced by carbon tetrachloride. Arch Pathol 57:1-11 